\title{
Synthetic aperture phase-shifting interferometry for high-numerical-aperture spherical surface measurement
}

\author{
Toshiki Kumagai $\odot,{ }^{\text {a,b,* } * \text { Yasunari Nagaike, }}{ }^{\text {a }}$ Kenichi Hibino, ${ }^{\mathrm{c}}$ and \\ Katsumi Wasaki $\odot^{b}$ \\ aOlympus Corporation, Corporate Research and Development Manufacturing, Kamiina-gun, \\ Nagano, Japan \\ ${ }^{\mathrm{b}}$ Shinshu University, Faculty of Engineering, Wakasato, Nagano, Japan \\ 'National Institute of Advanced Industrial Science and Technology, Tsukuba, Ibaraki, Japan
}

\begin{abstract}
In testing of high-numerical-aperture spherical lenses, conventional Fizeau interferometry with a mechanical phase modulator suffers from spatial nonuniformity of the phase steps within the observation aperture. The amount of phase modulation decreases rapidly in the marginal region of the aperture due to a geometrical effect, which results in a systematic error of several nanometers in the measured object profile. We propose a synthesis of phase-shifting algorithms in which several algorithms designed for different phase steps operate on the same set of interference fringes recorded in a single phase-shift sequence. Then, the object phase is determined from each of the algorithms for each aperture division. The resultant phase distribution showed minimal systematic errors. () The Authors. Published by SPIE under a Creative Commons Attribution 4.0 Unported License. Distribution or reproduction of this work in whole or in part requires full attribution of the original publication, including its DOI. [DOI: 10.1117/1.OE.60.1.014101]
\end{abstract}

Keywords: phase measurement; spherical testing; phase-shift nonuniformity; synthetic aperture; Fizeau interferometer.

Paper 20201222 received Oct. 15, 2020; accepted for publication Dec. 7, 2020; published online Jan. 4, 2021.

\section{Introduction}

High-numerical-aperture (NA) spherical surfaces are required for wide-angle and highmagnification industrial products, such as endoscopes, microscopes, and cameras, for metrology references in optical interferometers for aspherical lens testing and for coordinate measuring machines. Recently, spherical lenses have attained surface measurement repeatability by spherical Fizeau interferometers of better than 0.1-nm RMS. To further improve the repeatability and accuracy of high-NA spherical surface measurement, both random noise and systematic errors of the interferometers must be decreased. For the suppression of random noise, we have proposed a dual phase-shift scheme for Fizeau interferometers to minimize noise from the internally scattered light. ${ }^{1}$

Spherical Fizeau interferometers have a number of systematic error sources, ${ }^{2}$ including nonuniformity ${ }^{3}$ and nonlinearity ${ }^{4}$ in the phase modulation, multiple reflections, residual aberrations of converging optics, unstable source laser intensity, ${ }^{5}$ and air turbulence. Among these error sources, spatial nonuniformity of the phase shift is common in mechanical phase modulation. When a phase shift is introduced mechanically by a piezoelectric transducer (PZT), the phaseshift increment decreases by a cosine factor in the marginal region of the observation aperture, which is a purely geometrical effect.

Wavelength tuning ${ }^{5-9}$ is another option for high-NA spherical measurements because the amount of phase shift is proportional to the air gap distance. This technique can achieve a spatially uniform phase shift, but its accuracy suffers when the intensity of the light source varies, which needs to be addressed.

*Address all correspondence to Toshiki Kumagai, t_kumagai@ot.olympus.co.jp 
Iterative methods ${ }^{10-13}$ can detect not only the object phase value but also each phase shift value or phase modulation value due to unexpected vibrations and feedback to the object phase value using the spatial movement information, as well as the temporal variation of the interference fringes. The iterative models were formulated first with a two-beam interferometer ${ }^{10,11}$ and later with a white-light interferometer ${ }^{12}$ and spherical Fizeau cavity. ${ }^{13}$ The drawback of this technique is that the Fourier filtering of signals with higher harmonics is not appropriate, so that the number of parameters increases in multiple-beam interferometry.

A number of phase-shifting algorithms have been proposed that can compensate for the phase-shift increment errors. ${ }^{14-19}$ When the increment error becomes large, a coupling error also occurs between the harmonic signals (or multiple-reflection light beams inside the Fizeau cavity) and the phase-shift error. ${ }^{20}$ Typically, when the NA of the test surface is larger than 0.8 and the phase increment error falls below $-30 \%$, these coupling errors become substantial (larger than $1 \mathrm{~nm}$ ). With conventional techniques, multiple reflections can be suppressed by coating the reference surface with a partially absorbing thin film. ${ }^{21}$ However, the additional coating generally degrades the sphericity of the reference sphere. Kim et al. ${ }^{22,23}$ proposed $4 N-3$-frame and $6 N-5$-frame algorithms to compensate for couplings of higher harmonics up to the $N-2^{\text {nd }}$ order, where $N$ is the divisor of the phase-shift increment. These algorithms can be used for a reflecting surface with a reflectivity higher than that of glass. However, these algorithms need to modulate the interference signal for more than three or four periods, which introduces additional deviations of the test surface from a confocal position, causing additional spherical aberrations.

In this paper, we propose a phase determination over the entire observation aperture that, instead of a single phase-shifting algorithm, uses several algorithms designed for different phase increments. ${ }^{24}$ All the algorithms share the same set of interference images recorded with a single phase-shifting sequence. The amount of phase modulation introduced by a mechanical phase shifter decreases approximately proportional to $\cos \theta$, where $\theta$ is the angle between the illuminating beam and the optical axis. We divide the circular observation aperture into several annular regions and prepare an algorithm designed for a phase increment of $2 \pi / N^{\prime}$ radians for each region, where $N^{\prime}$ is the integer nearest the actual phase increment at the position. As an example, a spherical concave glass surface of $0.86 \mathrm{NA}$ was compared to a reference transmission spherical concave surface with the same NA. We divided the observation aperture into seven annular regions and prepared seven algorithms with different phase increments with divisors $N^{\prime}$ distributed from 6 to 12. The phase-shift increment on the optical axis was equal to $\pi / 3 \mathrm{rad}$ (a translation of $\lambda / 12$ ), while the corresponding increment at the marginal region was $\sim \pi / 6 \mathrm{rad}$. The reference surface was translated stepwise 12 times, and 13 interference images were recorded during the translation. The object phase distributions for the seven regions were then calculated and finally synthesized to undergo phase unwrapping.

The phase increment error or detuning error for the test surface of 0.86 NA was originally $-49 \%$ at the marginal region. The present synthetic technique can make the phase-increment parameter flexible and thus decrease the detuning error to less than $-8 \%$. Because the different algorithms have different susceptibilities to the detuning error, the calculated phase is expected to have a discontinuity at the boundary of each region. We evaluate the phase discontinuity at the boundary and also discuss the residual errors.

\section{Synthesis of Phase-Shifting Algorithms in a Fizeau Interferometer}

\subsection{Annular Sub-Aperture Division in a Spherical Fizeau Interferometer}

Figure 1 shows the optical setup of our Fizeau interferometer. The source was a stabilized 633-nm He-Ne laser. The output from the source was transmitted through a rotating groundglass diffuser and a multi-mode fiber $200 \mu \mathrm{m}$ in diameter to reduce the lateral coherence of the beam. The output beam from the fiber was then collimated, transmitted through a polarizer, and reflected by a polarization beam splitter. The beam was transmitted through a quarter-wave plate, expanded by a relay lens to a diameter of $60 \mathrm{~mm}$, and collimated to illuminate both a reference transmission spherical surface and the surface of a test object. The reflections from both surfaces returned along the original path were transmitted through the polarization beam splitter and were 


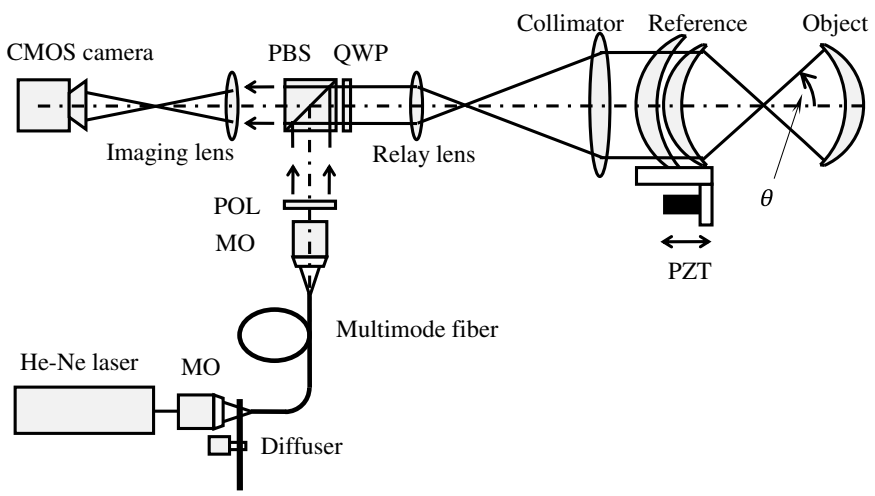

Fig. 1 Optical setup for the phase-shift Fizeau interferometer, where MO, POL, PBS, QWP, and PZT are the microscope objective, polarizer, polarization beam splitter, quarter wave plate, and piezoelectric transducer, respectively.

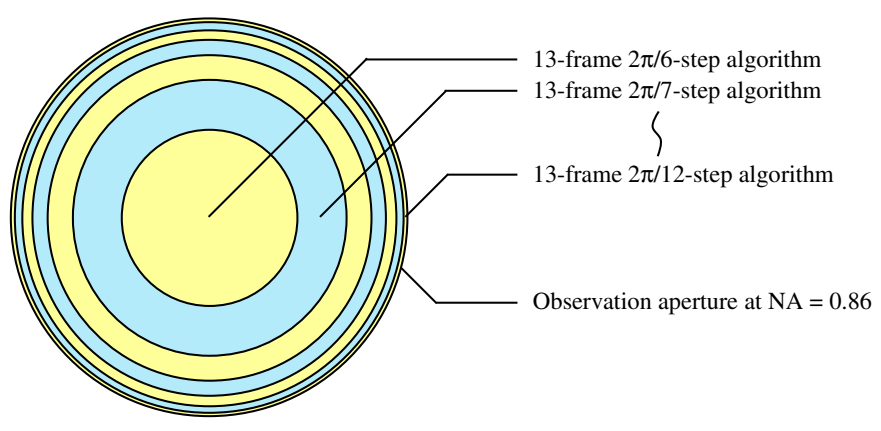

Fig. 2 Annular sub-aperture division in the observation aperture on the test object and the corresponding phase-shift increments for each region.

combined to form interference fringes on a CMOS camera $(1024 \times 1024$ pixels). The test and reference surfaces were positioned horizontally. The reference surface was translated along the optical axis by a PZT to introduce phase modulation. During the phase modulation, 13 interference images were recorded at equal time intervals. The relative phase step between frames was designed to be $60^{\circ}$ at the center of the aperture. The object phase was calculated by 7 different 13-frame phase-shifting algorithms, as described in Sec. 2.2.

Next, we discuss the detuning error, which depends on the NA in the spherical measurement. Figure 2 shows a schematic diagram of the division of the observation aperture on the test object. We divided the circular aperture of 0.86 NA into seven annular regions. The boundary between two neighboring regions was chosen to make the phase-detuning error coefficients equal along this boundary (see Sec. 2.3 for a detailed description of the boundary position.) The effective phase-shift increment produced by the mechanical PZT decreases by a factor of $\cos \theta$, where $\theta$ is the angle between the illuminating beam and the optical axis at the position. When the original phase-shift increment is $\pi / 3 \mathrm{rad}$ ( or $60^{\circ}$ ) on the optical axis, the increment at the outermost annular region of the aperture decreases to $(\pi / 3) \cos \theta \approx \pi / 6 \mathrm{rad}$. The detuning is therefore $-49 \%$ at maximum. After the division, we assigned a phase-shift increment of $2 \pi /(5+i)$, for region number $i=1,2, \ldots, 7$, to each region. After these assignments, the maximum detuning error of the phase modulation decreased from $-49 \%$ to $-7.7 \%$.

\subsection{3-Frame 2 $\pi / N$-Step Phase-Shift Algorithms for Phase Measurement}

Here, we describe the sampling weights of the seven algorithms used for measurement and briefly define the notation. We confine our discussion to a single sub-aperture region where the divisor of the phase-shift increment is defined by integer $N$, although it is applicable to all 
other regions. The object phase in the region is a function of position $(x, y)$ on the observation aperture and is calculated by an $M$-frame algorithm as

$$
\varphi=\arctan \left[\sum_{r=1}^{M} b_{r} I\left(\alpha_{r}\right) / \sum_{r=1}^{M} a_{r} I\left(\alpha_{r}\right)\right],
$$

where $a_{r}$ and $b_{r}$ are the $r^{\prime}$ th sampling amplitudes, $I\left(\alpha_{r}\right)$ is the fringe intensity, and $\alpha_{r}$ is the phase shift. If we define the sampling window as $w_{r}$, the sampling amplitudes are denoted by

$$
a_{r}=w_{r} \cos \alpha_{0 r}
$$

and

$$
b_{r}=w_{r} \sin \alpha_{0 r}
$$

The phase-shift value for the $N$-division region can be described with error coefficient $\varepsilon_{i}$ by

$$
\alpha_{r}=\alpha_{0 r}\left[1+\varepsilon_{1}+\varepsilon_{2}\left(\frac{\alpha_{0 r}}{T}\right)+\varepsilon_{3}\left(\frac{\alpha_{0 r}}{T}\right)^{2}+\cdots\right]
$$

where the unperturbed phase shift is defined by

$$
\alpha_{0 r}=\frac{2 \pi}{N}\left(r-\frac{M+1}{2}\right),
$$

and the half-width of the total phase shift is defined by

$$
T=\frac{\pi}{N}(M-1)
$$

The phase-shift errors are caused by nonlinearity of the PZT response and the spatial nonuniformity of the mechanical phase shift. Because the algorithm is designed for a phase-shift increment of $2 \pi / N$ rad, while the actual increment expected from the geometry is $(\pi / 3) \cos \theta$, the first-order error coefficient $\varepsilon_{1}$ approximately equals

$$
\varepsilon_{1}=\left(\frac{\pi \cos \theta}{3}-\frac{2 \pi}{N}\right) /\left(\frac{2 \pi}{N}\right)=\frac{N}{6} \cos \theta-1, \quad \text { for } N=6,7,8, \ldots, 12,
$$

where $\theta$ is the angle between the illuminating beam (or surface normal) and the optical axis at the position. In practical experiments, the coefficient $\varepsilon_{1}$ also includes the gain error of the PZT.

The measurement error in the calculated phase $\Delta \varphi=\varphi-\varphi_{0}$ is a function of these error coefficients and the object phase $\varphi_{0}$, and can be expanded using coefficients $J_{i}, K_{i}$, and $F_{i}$ as

$$
\Delta \varphi=J_{1} \varepsilon_{1} \sin 2 \varphi_{0}+K_{0} \varepsilon_{2}+K_{1} \varepsilon_{2} \cos 2 \varphi_{0}+F_{1} R \varepsilon_{1} \sin \varphi_{0}+F_{2} R \varepsilon_{1} \sin 3 \varphi_{0} .
$$

Table 1 shows the sampling weights $w_{r}$ of the seven algorithms for $N=6,7, \ldots, 12$. All algorithms are designed to share 13 interference images $(M=13)$. The first algorithm with divisor $N=6$ is the algorithm we used in the random noise suppression, as discussed in Ref. 1 . The last algorithm with divisor $N=12$ is identical to the Schwider-Hariharan 5-frame algorithm, ${ }^{2,25}$ which has a phase increment designed for $\pi / 2$ rad. The other five windows with divisors $N=7,8, \ldots, 11$ were created by a convolution of three windows, as described later. Convolutions of rectangular windows generally reduce the cross-talk from the harmonic signals ${ }^{26}$ and thus are expected to reduce the magnitudes of error coefficients $F_{1}$ and $F_{2}$ in Eq. (8).

We started with a convolution between $N$-frame and $12-N$-frame rectangular windows to produce an 11-frame trapezoidal window $B=\left(b_{1}, \ldots, b_{11}\right)$. The trapezoidal window was then convoluted with a 3 -frame window $G_{N}=\left(1, g_{N}, 1\right)$ to generate a final 13-frame window, where parameter $g_{N}$ is defined by 


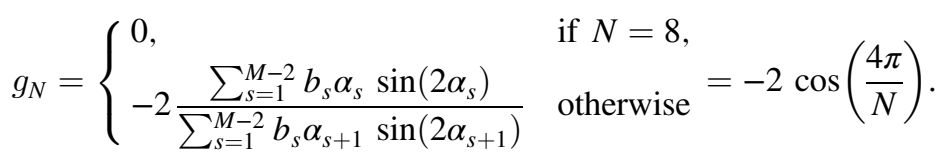

This parameter was chosen so that coefficient $J_{1}$ becomes 0 [see Eq. (8)] ${ }^{18}$ In the case of $N=8$, because the $J_{1}$ coefficient is inherently 0 , the parameter $g_{N}$ was chosen to have a coefficient of 0 for $K_{1}$.

As an example, we follow the calculation of a window with $N=8$. We first took a convolution of an 8-frame rectangular window with the 4-frame one to produce an 11-frame trapezoidal window, which was calculated to be

$$
(1,1,1,1) *(1,1,1,1,1,1,1,1)=(1,2,3,4,4,4,4,4,3,2,1),
$$

where the asterisk (*) denotes a convolution. Finally, we convoluted the trapezoidal window and the ancillary window $G_{8}=(1,0,1)$ and obtained the 13 -frame window in Table 1 as

$$
(1,0,1) *(1,2,3,4,4,4,4,4,3,2,1)=(1,2,4,6,7,8,8,8,7,6,4,2,1) .
$$

The frequency transfer function (FTF) of the algorithm can visualize the Fourier filtering specification and impart robustness to the phase-shift error. The FTF is defined by ${ }^{27}$

$$
H_{N}(\nu)=\sum_{r} w_{r} \exp \left\{-i \alpha_{0 r}\left(\nu / \nu_{0}-1\right)\right\}
$$

where $\nu_{0}$ is the sampling frequency of the image acquisition. Figure 3 shows the FTFs for the algorithms with (a) $N=6$, (b) $N=7$, (c) $\mathrm{N}=8$, (d) $N=9$, (e) $N=10$, (f) $N=11$, and (g) $N=12$, whose sampling weights are given in Table 1 . The functions are zero at negative frequency $\nu / \nu_{0}=-1$ and in its vicinity, which shows robustness against the phase increment error. All algorithms except for the seventh have no sensitivity to harmonic frequencies of $2 \nu_{0}$ to $4 \nu_{0}$, which can filter out the multiple-beam interference noise.

The sensitivity to random additive noise for the algorithm is estimated by a signal-to-noise ratio (SNR) gain ${ }^{28}$ (also called the "process" gain ${ }^{29}$ ). It is a ratio of the coherent signal gain to incoherent noise gain, and is defined in terms of the response function and the weight by

$$
S N R \text { gain }=\frac{\left|H\left(v_{0}\right)\right|^{2}}{\int_{-N v_{0} / 2}^{N v_{0} / 2}|H(v)|^{2} \mathrm{~d} v}=\frac{\left(\sum_{r} w_{r}\right)^{2}}{\sum_{r} w_{r}^{2}} .
$$

Table 1 Sampling windows for the seven phase-shifting algorithms used during measurement and their SNR gain values.

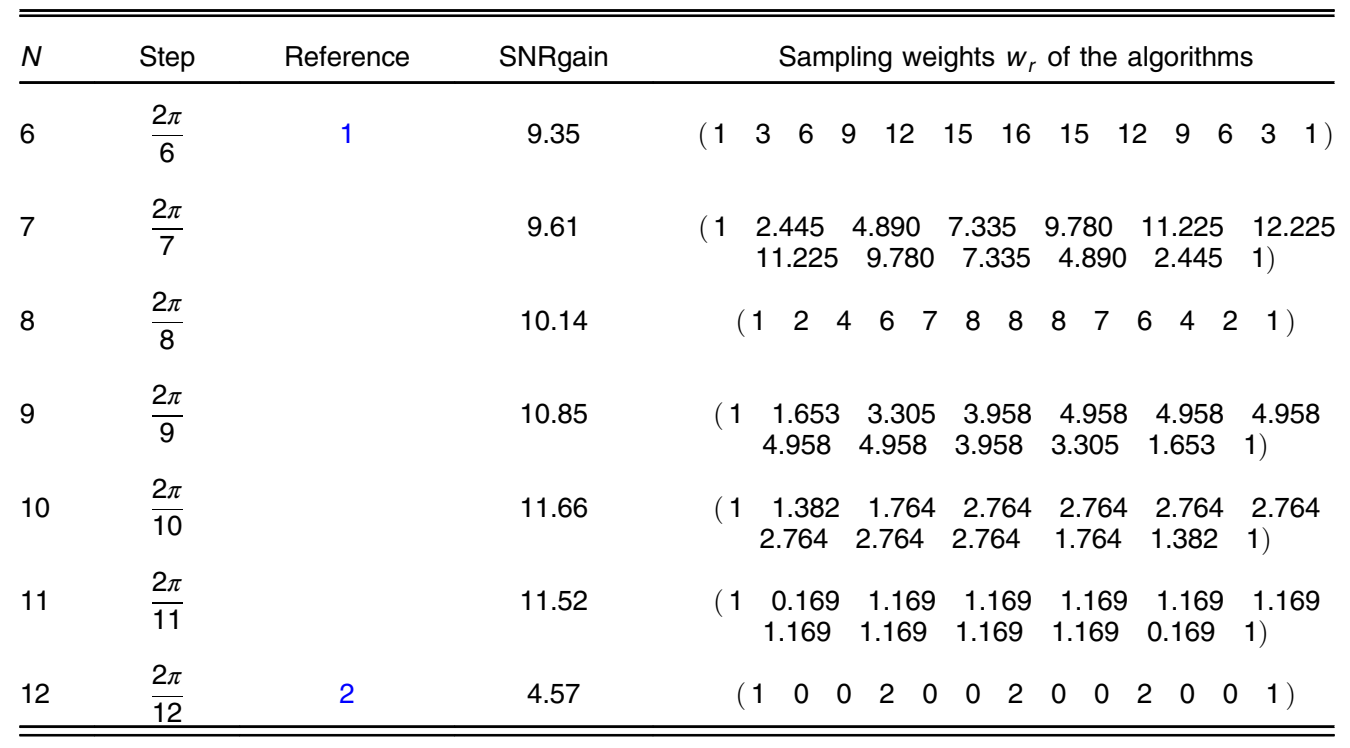




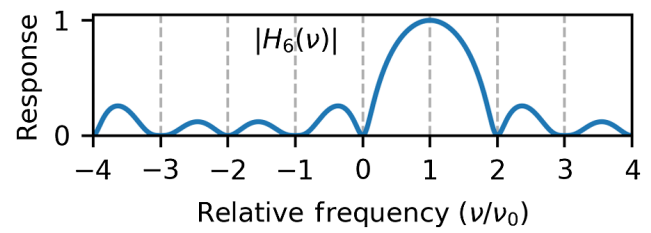

(a)

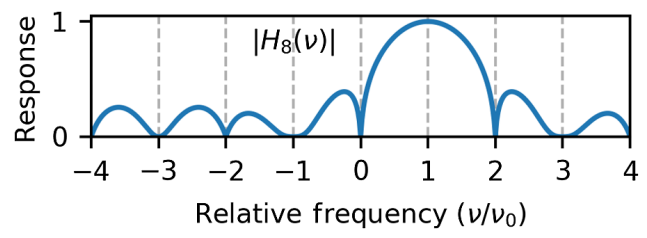

(c)

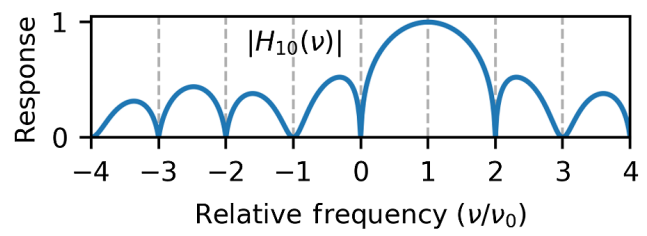

(e)

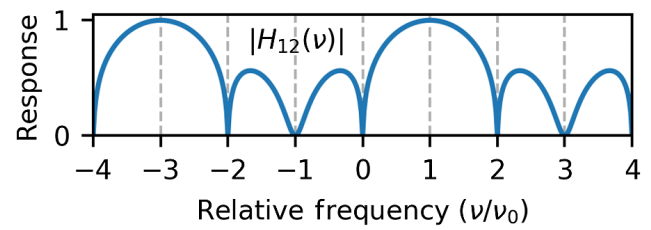

(g)

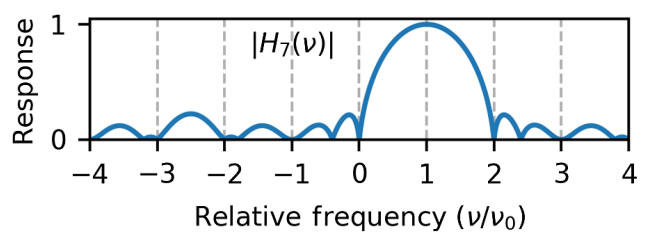

(b)

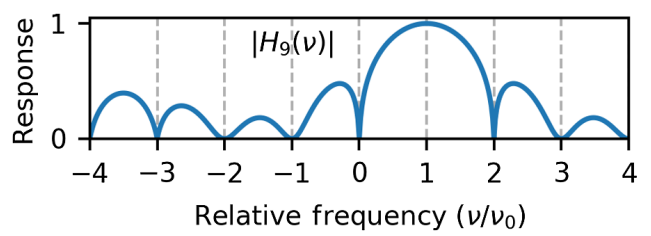

(d)

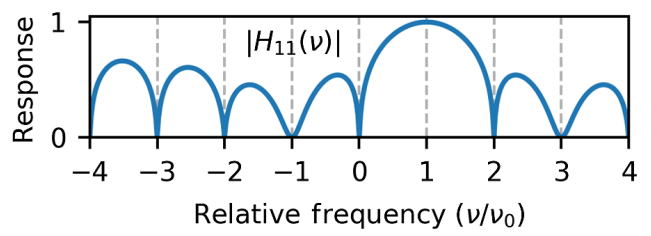

(f)

Fig. 3 FTFs of the seven algorithms, where the sampling weights are shown in Table 1: (a) $N=6$, (b) $N=7$, (c) $N=8$, (d) $N=9$, (e) $N=10$, (f) $N=11$, and (g) $N=12$. The ordinate is compressed as $(1 / 2) \log _{10}[|H(\nu)|+1]$, where the sampling weights are normalized with $\Sigma w_{r}=99$ for clarity of viewing.

Table 1 also shows the SNRgain for the seven algorithms. The maximum SNRgain equals 13 (frame number $M$ ) when the sampling window is rectangular. In the seventh algorithm $(N=12)$, the SNRgain yields a poor value because only five samples are used.

\subsection{Numerical Calculation of the Phase Measurement Errors}

Here, we numerically evaluate the phase measurement errors during measurement testing of a spherical glass surface of $0.86 \mathrm{NA}$ and reflection index $R_{1}=3.5 \%$ and compare it with a reference transmission surface of $R_{2}=7.9 \%$. The irradiance signal of the Fizeau interferometer is given $^{30}$ by

$$
I(\alpha)=I_{0}\left[1-\frac{\left(1-R_{1}\right)\left(1-R_{2}\right)}{1+R_{1} R_{2}-2 \sqrt{R_{1} R_{2}} \cos \left(\alpha-\varphi_{0}\right)}\right],
$$

where $R_{1}$ and $R_{2}$ are the reflection indices of the test and reference surfaces, respectively, $\varphi_{0}$ is the object phase to be measured, and $\alpha$ is the phase shift parameter. For simplicity, we assume that the phase-shift error is only caused by the geometrical effect of the NA and neglect the nonlinearity of the PZT. The error coefficient $\varepsilon_{1}$ for each sub-aperture region is then simply given by Eq. (7). The phase shift at each position in the observation aperture is reduced to 
Kumagai et al.: Synthetic aperture phase-shifting interferometry for high-numerical-aperture ...

Table 2 Sampling windows for the five conventional phase-shifting algorithms.

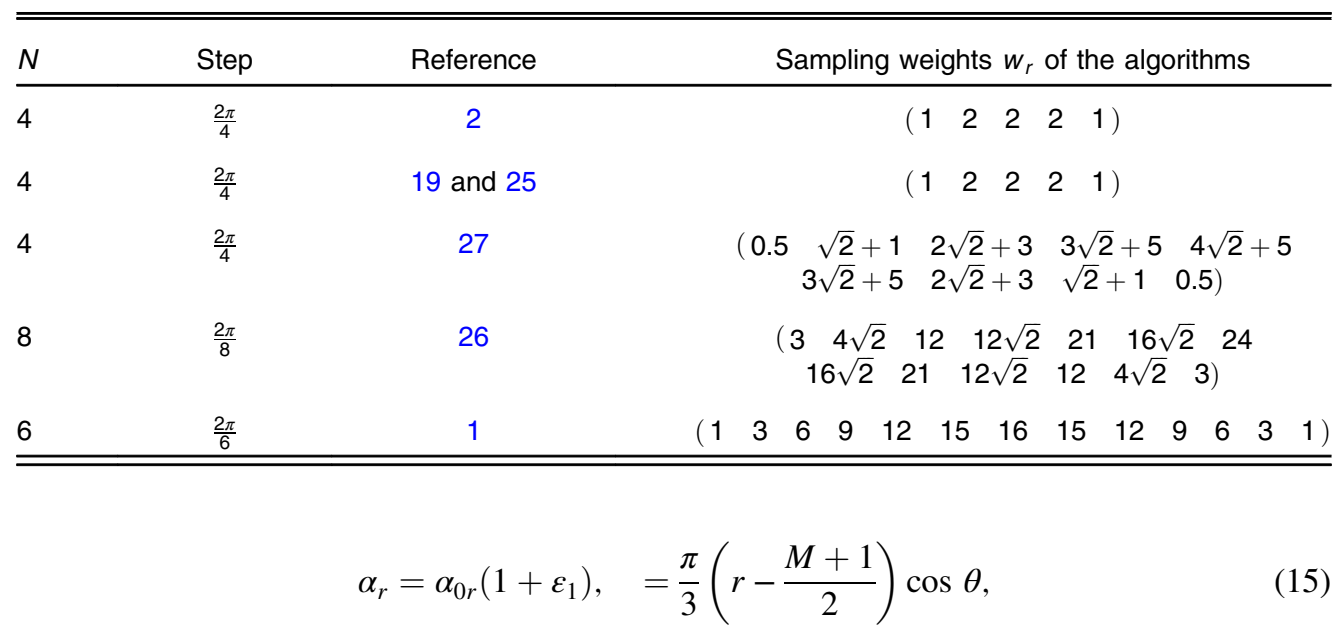

where $\sin \theta$ is the NA at each position and is a function of the coordinates $(x, y)$.

First, we evaluated the phase measurement errors in the conventional techniques, that is, we calculated the object phase over the whole aperture with only a single algorithm. We used five conventional algorithms: Schwider-Hariharan 5-frame, ${ }^{2,25}$ Hariharan-Novak adaptive 5frame, ${ }^{19,25}$ de Groot 13 -frame, ${ }^{26}$ Servin 9-frame, ${ }^{27}$ and Kumagai 13 -frame. ${ }^{1}$ The corresponding sampling windows and phase-shift increments are shown in Table 2. The Hariharan-Novak algorithm is defined by

$$
\varphi=\arctan \left[\frac{2\left(I_{2}-I_{4}\right)}{I_{1}-2 I_{3}+I_{5}} \sin \psi\right],
$$

where $\psi=(\pi / 2) \cos \theta$ is an adaptive correction factor depending on the position and equals the real phase-shift increment.

The phase measurement error $\varphi-\varphi_{0}$ is a function of the object phase $\varphi_{0}$ [see Eq. (8)]. We therefore define the standard deviation of the error by averaging the square of the error over the $2 \pi$ period as

$$
\overline{\Delta \varphi}=\sqrt{\frac{1}{2 \pi} \int_{0}^{2 \pi}\left(\varphi-\varphi_{0}\right)^{2} \mathrm{~d} \varphi_{0}} .
$$

Figure 4 shows the standard deviations of errors as a function of the phase-shift error $\varepsilon_{1}=\cos \theta-1$ for the central region and the corresponding $\mathrm{NA}=\sin \theta$. It can be observed that the phase errors are small as long as the NA is smaller than 0.6; however, they increase rapidly when the NA approaches 0.85 . The maximum error is around $4 \mathrm{~nm}$ with a single algorithm.

Second, we calculated the phase error for the present synthetic technique by applying the seven algorithms shown in Table 1 to the intensity signal of Eq. (14) with the phase shift of Eq. (15). Figure 5 shows the overlapped standard deviations of the phase errors for the seven algorithms as functions of $\mathrm{NA}=\sin \theta$ and $\varepsilon_{1}=\cos \theta-1$. We can observe that the errors increase very rapidly, especially when the NA is high.

Now, we discuss the sub-aperture division. The intersection between every pair of neighboring error curves in Fig. 5 indicates the optimum position of the boundary for dividing the aperture into sub-aperture regions. However, the actual error curves are also affected by the PZT nonlinearity. For simplicity of calculation, we define the boundary as the position along which the error coefficients $\varepsilon_{1}$ of the two regions are equal. To be more specific, the first-order error coefficients for the divisor- $N$ and divisor- $N+1$ regions are equal in magnitude along their boundary as described by

$$
\varepsilon_{1, N}=-\varepsilon_{1, N+1}
$$




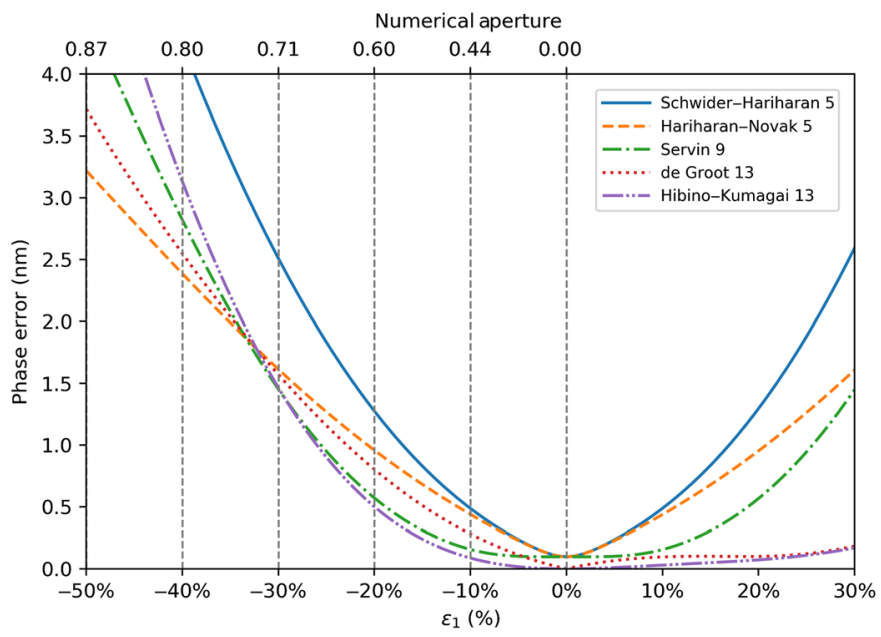

Fig. 4 Standard deviations of the phase measurement errors in for a spherical surface of 0.86 NA and $R_{1}=3.5 \%$, compared with a reference surface of $R_{2}=7.9 \%$ as functions of the phase-shift error and corresponding NA calculated by five conventional algorithms: Schwider-Hariharan 5frame, Hariharan-Novak 5-frame, Servin 9-frame, de Groot 13-frame, and Kumagai 13-frame.

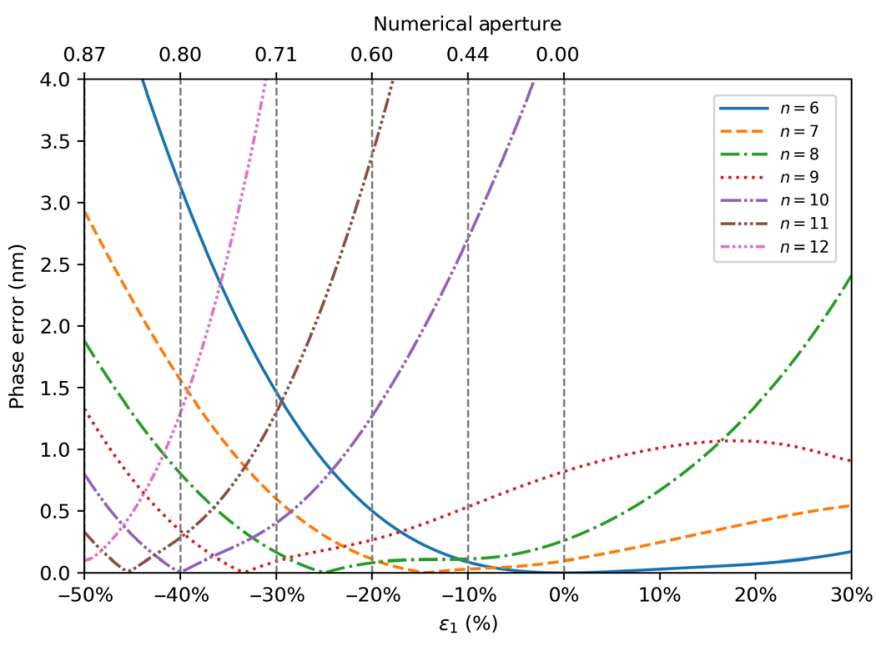

Fig. 5 Overlapped standard deviations of the phase measurement errors for the seven algorithms as functions of $\mathrm{NA}=\sin \theta$ and $\varepsilon_{1}=\cos \theta-1$.

where we note that the signs of the error coefficients are opposite. After the coefficient values of Eq. (7) are substituted into Eq. (18), this condition can be rewritten as

$$
\frac{N}{6} \cos \theta-1=1-\frac{N+1}{6} \cos \theta \text {. }
$$

The boundary position for the sub-aperture region with divisor $N$ is then defined by

$$
\cos \theta=\frac{12}{2 N+1}, \quad \text { for } N=6,7, \ldots, 11
$$

The resultant sub-aperture division based on Eq. (20) is shown in Fig. 2.

We then addressed the object phases corresponding to the sub-aperture division shown in Fig. 2. Figure 6 shows the resultant phase errors by the present synthetic method. We can observe that the maximum standard deviation of the error caused by the spatial nonuniformity of the phase shift is decreased from 4 to $0.2 \mathrm{~nm}$ or $1 / 20$ th of that caused by a single algorithm. 


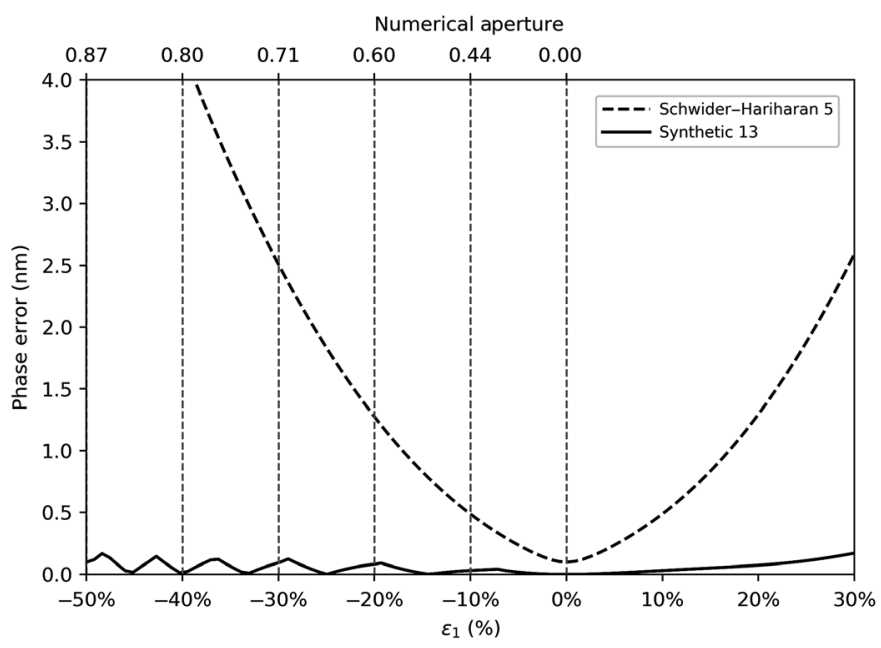

Fig. 6 Standard deviation of the error in measurement of a spherical surface of 0.86 NA and $R_{1}=3.5 \%$, compared with a reference surface of $R_{2}=7.9 \%$ by the synthetic method.

\section{Experiments}

A spherical concave glass surface with $17-\mathrm{mm}$ diameter, $R=3.5 \%$, and $0.86 \mathrm{NA}$, shown in Fig. 7, was compared to a reference transmission concave surface with $38-\mathrm{mm}$ diameter, $R=7.9 \%$, and the same NA. The optical setup of the measurement system is shown in Fig. 1. The reference surface was translated along the optical axis by a PZT to introduce phase modulation. Thirteen interference images were recorded with an equal phase-shift increment of $\pi / 3 \mathrm{rad}$. The object phases were calculated by the seven different algorithms discussed in Sec. 2.2. The synthetic object phase was calculated by determining the result of the phase calculation in each of the regions shown in Fig. 2.

As a comparison, the object phase over the entire aperture was calculated by the single algorithm shown in Table 1 for $N=6$. Figures 8 (a) and 8(b) show the object phases calculated by the single algorithm. The alignments of the objects in Figs. 8(a) and 8(b) were the null condition and slightly tilted in the horizontal direction, respectively. In each panel, the raw interferogram is shown in the right bottom corner. From Eq. (8), we know that the systematic error caused by the phase-shift error depends on the object phase $\varphi_{0}$. Therefore, if we introduce a slight tilt modulation in the object phase, we can observe a similar modulated error in the final object phase. In Fig. 8(b), we can observe a systematic error with an apparent magnitude of $6 \mathrm{~nm}$ in the marginal region. From this result, we can see that the phase measurement by a conventional single algorithm suffers from a significant phase error.

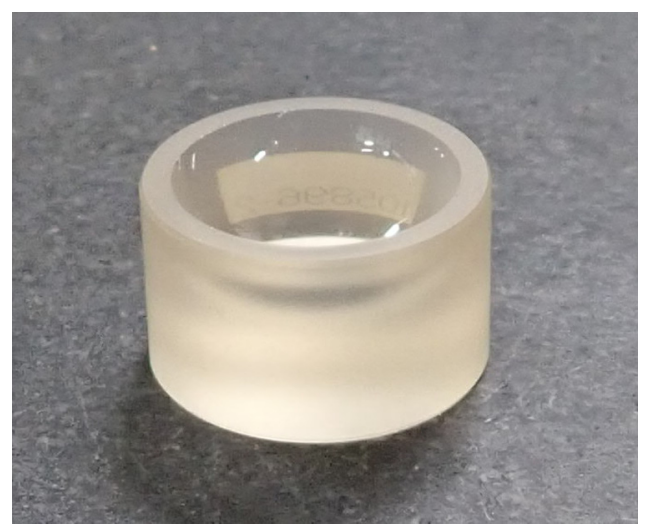

Fig. 7 Spherical concave test object $17 \mathrm{~mm}$ in diameter, with a 10-mm radius of curvature $(\mathrm{NA}=0.86)$. 


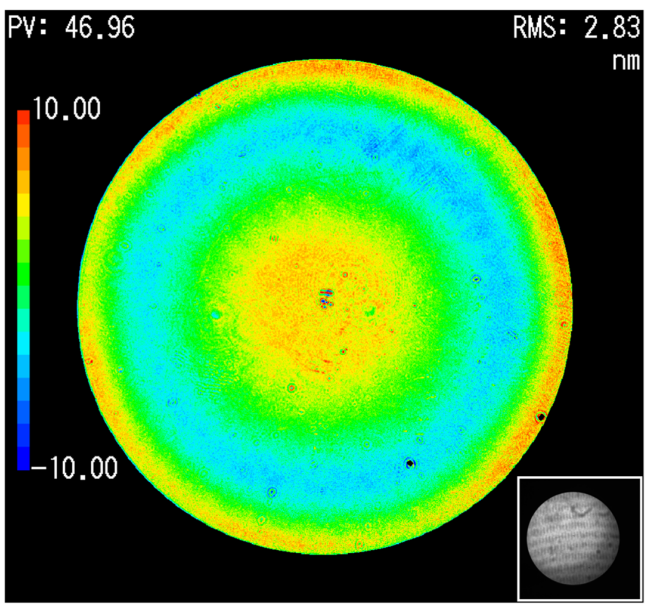

(a)

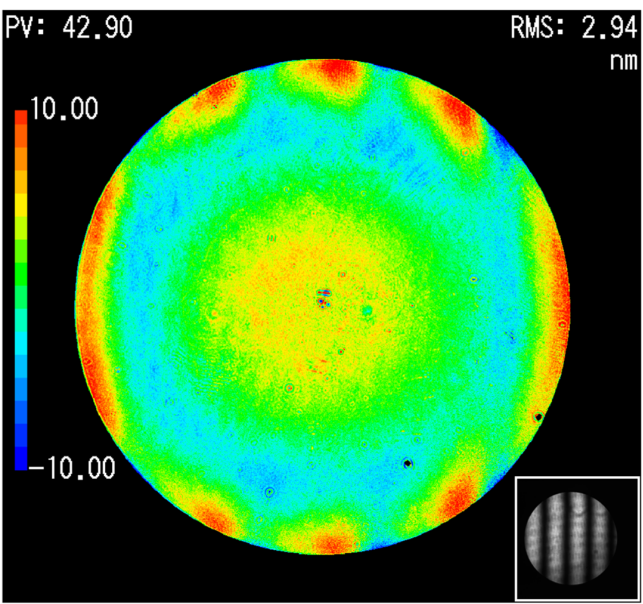

(b)

Fig. 8 Measured phase distributions of a 0.86 NA spherical surface obtained by a single 13-frame algorithm (after removing tilt and defocus): (a) null alignment and (b) slightly tilted in the horizontal direction.

Figures 9(a) and 9(b) show the object phases calculated by the present synthetic method using the same set of 13 interference images as in Figs. 8(a) and 8(b), respectively. Comparing the results of Figs. 8(a) and 8(b), we can see that the calculated phases do not depend on the initial tilt bias of the phase. We estimate the magnitude of the systematic error to be less than $1 \mathrm{~nm}$, which is much smaller than the result shown in Fig. 8(b). From these results, we conclude that the present synthetic method can significantly suppress phase errors caused by spatial nonuniformity of the phase modulation in high-NA spherical measurements.

Finally, we discuss the residual systematic error that is characteristic of the present synthetic method. By subtracting the phase of Fig. 9(a) from the phase of Fig. 9(b), we can observe a systematic error that depends on the phase $\varphi_{0}$. Figure 10 shows the difference phase after removing the 36 Zernike components. In the figure, the broken lines show the boundaries of the seven annular regions. We can observe discontinuous phase gaps of approximately $1 \mathrm{~nm}$ along a couple of boundaries. On opposite sides of each boundary, the error coefficient $\varepsilon_{1}$ has a different sign. For example, at the boundary between the regions for $N=11$ and $N=12$ [see Eq. (7)], the

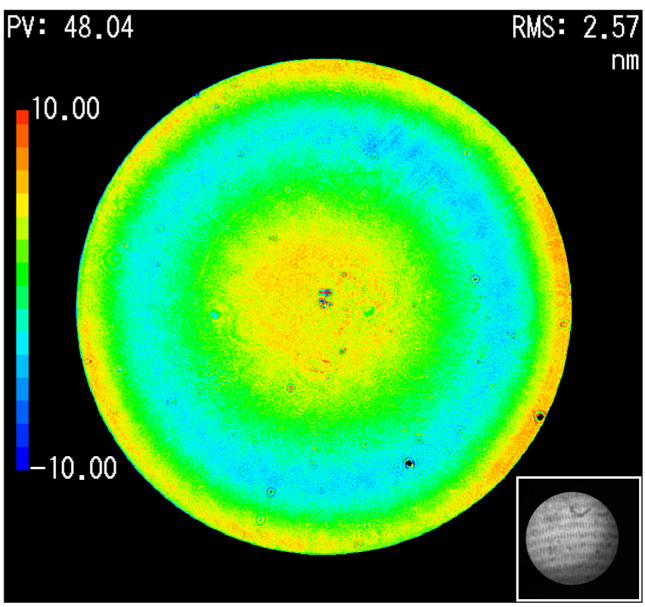

(a)

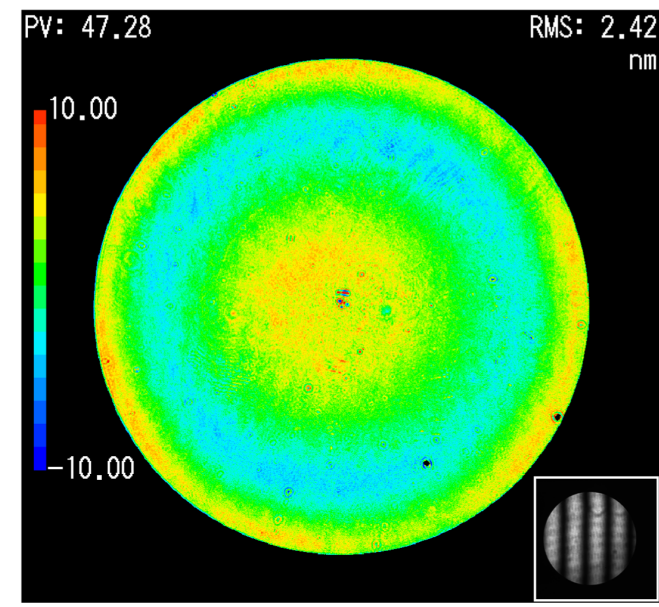

(b)

Fig. 9 Measured phase distributions of a 0.86 NA spherical surface obtained by the seven algorithms using the same interference images of Fig. 8 (after removing tilt and defocus): (a) null alignment and (b) slightly tilted in the horizontal direction. 


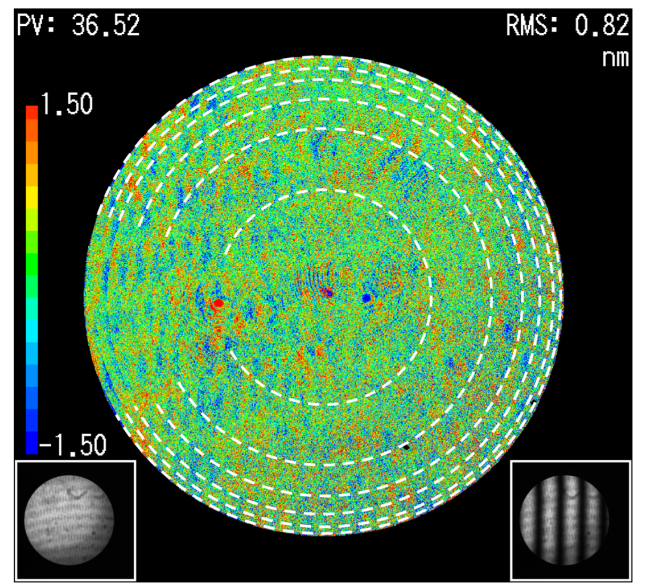

Fig. 10 Phase difference between Figs. 9(a) and 9(b) that depends on the object phase after removing the 36 Zernike components.

coefficients for both ends have similar magnitudes but different signs $\left(\varepsilon_{1}= \pm 0.043\right)$. Then, the resultant phase-shift errors for both sides caused by the coefficient $\varepsilon_{1}$ are also expected to have different signs, which appeared in the phase gap in the residual errors of Fig. 10. The different signs can cause a discrete change in the correlated errors, including $\varepsilon_{1} R$ [see Eq. (8)].

We also need to address the nonlinear response of the PZT. The PZT used in the present study was an open-loop type, which typically has a nonlinear response of $2 \%\left(\varepsilon_{2}=0.02\right)$. However, the error coefficient $K_{0}$ in the second term of Eq. (8) has a magnitude of several tens of nanometers and varies greatly from algorithm to algorithm. The nonuniformity in $K_{0}$ can also cause a discrete change in the phase error. It is considered that these two effects appeared in the phase gap in the residual errors of Fig. 10. The sign difference cannot be seen directly in the error variation of Fig. 5, because this figure shows only the error magnitude.

\section{Conclusions}

In spherical Fizeau interferometers with mechanical phase modulation, spatial nonuniformity in the phase-shift increment within the observation aperture causes a systematic error in the measured phase. In particular, error-compensating phase-shifting algorithms do not provide sufficient compensation for the error in high-NA spherical surface tests. We propose a synthesis of phase-shifting algorithms in which the observation aperture is divided into several annular regions and the object phase for each region is calculated by a different algorithm. The algorithms are designed with different ideal phase steps, but they can operate on the same set of interference fringes that are recorded during an ordinary single measurement. Therefore, the measurement time necessary for a single measurement is similar to that for an ordinary phase-shift measurement. A spherical concave surface of 0.86 NA was compared to a reference transmission concave surface. The observation aperture was divided into seven annular regions, each with a different algorithm, in phase steps of $2 \pi / N$ rad, where the integer $N$ is distributed from 6 to 12. Selecting these different phase steps decreased the spatial nonuniformity of the phase increment from $49 \%$ to $7.7 \%$. The resultant systematic error in the measured phase decreased to less than $1 \mathrm{~nm}$. Because the sensitivity of the algorithm to the phase-shift error differs depending on the algorithm, a discontinuous error of approximately $1 \mathrm{~nm}$ was observed at each border between neighboring regions.

\section{Acknowledgments}

This research was funded by Olympus Corporation, Nagano, Japan. The authors declare that they have no conflicts of interest related to publication of this research. 


\section{References}

1. T. Kumagai, K. Hibino, and Y. Nagaike, "Dual-phase-shift spherical Fizeau interferometer for reducing the internally scattered light noise," Opt. Eng. 56(3), 034102 (2017).

2. J. Schwider et al., "Digital wavefront measuring interferometry: some systematic error sources," Appl. Opt. 22, 3421-3432 (1983).

3. K. Creath and P. Hariharan, "Phase-Shifting errors in interferometric tests with highnumerical-aperture reference surfaces," Appl. Opt. 33, 24-25 (1994).

4. C. Ai and J. C. Wyant, "Effect of piezoelectric transducer nonlinearity on phase shift interferometry," Appl. Opt. 26, 1112-1116 (1987).

5. Y. Ishii, J. Chen, and K. Murata, "Digital phase-measuring interferometry with a tunable laser diode," Opt. Lett. 12, 233-235 (1987).

6. G. E. Sommargren, "Interferometric wavefront measurement," US Patent No. 4594003 (1986).

7. K. Okada et al., "Separate measurements of surface shapes and refractive index inhomogeneity of an optical element using tunable-source phase shifting interferometry," Appl. Opt. 29, 3280-3285 (1990).

8. M. Suematsu and M. Takeda, "Wavelength shift interferometry for distance measurement using a Fourier transform technique for fringe analysis," Appl. Opt. 30, 4046-4055 (1991).

9. L. Deck, "Fourier-transform phase shifting interferometry," Appl. Opt. 42(13), 2354-2365 (2003).

10. K. Okada, A. Sato, and J. Tsujiuchi, "Simultaneous calculation of phase distribution and scanning phase shift in phase shifting interferometry," Opt. Commun. 84, 118-124 (1991).

11. Z. Wang and B. Han, "Advanced iterative algorithm for phase extraction of randomly phaseshifted interferograms," Opt. Lett. 29(14), 1671-1673 (2004).

12. F. Munteanu and J. Schmit, "Iterative least square phase-measuring method that tolerates extended finite bandwidth illumination," Appl. Opt. 48(6), 1158-1167 (2009).

13. L. Deck, "Model-based phase shifting interferometry," Appl. Opt. 53(21), 4628-4636 (2014).

14. K. G. Larkin and B. F. Oreb, "Design and assessment of symmetrical phase-shifting algorithms," J. Opt. Soc. Am. A 9, 1740-1748 (1992).

15. K. Hibino et al., "Phase shifting for nonsinusoidal waveforms with phase-shift errors," J. Opt. Soc. Am. A 12, 761-768 (1995).

16. P. de Groot, "Derivation of algorithms for phase-shifting interferometry using the concept of a data sampling window," Appl. Opt. 34, 4723-4730 (1995).

17. J. Schmit and K. Creath, "Extended averaging technique for derivation of error-compensating algorithms in phase-shifting interferometry," Appl. Opt. 34, 3610-3619 (1995).

18. Y. Surrel, "Design of algorithms for phase measurements by the use of phase stepping," Appl. Opt. 35, 51-60 (1996).

19. J. Novak, "Five-step phase-shifting algorithms with unknown values of phase shift," Optik 114, 63-68 (2003).

20. P. de Groot, "Correlated errors in phase-shifting laser Fizeau interferometry," Appl. Opt. 53, 4334-4342 (2014).

21. P. B. Clapham and G. D. Dew, "Surface-coated reference flats for testing fully aluminized surfaces by means of the Fizeau interferometer," J. Sci. Instrum. 44, 899-902 (1967).

22. Y. Kim et al., "Multiple-surface interferometry of highly reflective wafer by wavelength tuning," Opt. Express 22(18), 21145-21156 (2014).

23. Y. Kim et al., "Simultaneous measurement of surface shape and optical thickness using wavelength tuning and a polynomial window function," Opt. Express 23(25), 3286932880 (2015).

24. T. Kumagai, Y. Nagaike, and K. Hibino, "Synthetic aperture phase-shifting interferometry for high numerical-aperture spherical surface measurement," Proc. SPIE 10749, 1074915 (2018).

25. P. Hariharan, B. F. Oreb, and T. Eiju, "Digital phase-shifting interferometry: a simple errorcompensating phase calculation algorithm," Appl. Opt. 26(13), 2504-2505 (1987). 
Kumagai et al.: Synthetic aperture phase-shifting interferometry for high-numerical-aperture ...

26. P. de Groot, "Measurement of transparent plates with wavelength-tuned phase-shifting interferometry," Appl. Opt. 39, 2658-2663 (2000).

27. M. Servin, J. C. Estrada, and J. A. Quiroga, "The general theory of phase shifting algorithms," Opt. Express 17(24), 21867-21881 (2009).

28. M. Servin, J. A. Quiroga, and J. M. Padilla, "Noise in temporal PSI," Chapter 2.7 in Fringe Pattern Analysis for Optical Metrology, p. 84, Wiley-VCH (2014).

29. J. Harris, "On the use of windows for harmonic analysis with the discrete Fourier transform," Proc. IEEE 66, 51-83 (1978).

30. G. Bonsch and H. Bohme, "Phase-determination of Fizeau interferences by phase-shifting interferometry," Optik 82(4), 161-164 (1989).

Toshiki Kumagai is an optical engineer at the Olympus Corporation, Department of Corporate Research and Development Manufacturing. His current work includes the development of optical interferometers and the design and measurement of optical components.

Yasunari Nagaike is an optical engineer at the Olympus Corporation, Department of Corporate Research and Development Manufacturing. His current work includes the development of measurement technology for optical components and systems.

Kenichi Hibino is a research scientist at the National Institute of Advanced Industrial Science and Technology. He received his BS and MS degrees in physics from the University of Tokyo in 1979 and 1981, respectively, and his PhD in engineering from the University of Tokyo in 1998. His current research interests include optical interferometers, holography, and length metrology.

Katsumi Wasaki received his MS and PhD degrees in computer science and engineering from Shinshu University in 1993 and 1997, respectively. He has been a professor in the Department of Computer Science and Engineering at Shinshu University since 1998. He has also worked with the University of Alberta at Edmonton and the New Energy and Industrial Technology Development Organization, Japan. His current research interests include modeling and analysis of parallel processing systems. 